\title{
Refined gauge group decompositions
}

\author{
D. Kishimoto, A. Kono, and S. Theriault
}

\begin{abstract}
Let $G$ be a simply connected, compact Lie group, let $P \longrightarrow S^{4}$ be a principal $G$-bundle, and let $\mathcal{G}(P)$ be the gauge group of this bundle. When $G$ is a matrix group and $p$ is an odd prime, we use new methods to improve on the $p$-local homotopy decompositions of $\mathcal{G}(P)$ appearing in separate work of the first two authors and the third author.
\end{abstract}

\section{Introduction}

Let $G$ be a simply connected, simple compact Lie group. Equivalence classes of principal $G$-bundles over $S^{4}$ are classified by the second Chern class, which can take any integer value. For $k \in \mathbb{Z}$, let $P_{k} \longrightarrow S^{4}$ be the principal $G$-bundle whose second Chern class has value $k$. The gauge group of $P_{k}$ is the group of $G$-equivariant automorphisms of $P_{k}$ which fix $S^{4}$; we label this as $\mathcal{G}_{k}(G)$.

In this paper we give a $p$-local homotopy decomposition of $\mathcal{G}_{k}(G)$ when $G$ is a matrix group and $p$ is an odd prime. This uses new methods to improve upon existing decompositions in [KKT] and [T1]. To describe this, we first relate the topology of Lie groups, gauge groups, and mapping spaces.

Let $B G$ and $B \mathcal{G}_{k}(G)$ be the classifying spaces of $G$ and $\mathcal{G}_{k}(G)$, respectively. Let $\operatorname{Map}\left(S^{4}, B G\right)$ and $\operatorname{Map}^{*}\left(S^{4}, B G\right)$, respectively, be the spaces of freely continuous and pointed continuous maps between $S^{4}$ and $B G$. The components of each space are in one-to-one correspondence with the integers, where the integer is determined by the degree of a map $S^{4} \longrightarrow B G$. By [G], there is a homotopy equivalence $B \mathcal{G}_{k} \simeq \operatorname{Map}_{k}\left(S^{4}, B G\right)$ between $B \mathcal{G}_{k}$ and the component of $\operatorname{Map}\left(S^{4}, B G\right)$ consisting of maps of degree $k$. Evaluating a map at the basepoint of $S^{4}$, we obtain a map ev: $B \mathcal{G}_{k} \stackrel{\text { ev }}{\longrightarrow} B G$ whose fiber is homotopy equivalent to $\operatorname{Map}_{k}^{*}\left(S^{4}, B G\right)$. It is well known that each component of $\operatorname{Map}^{*}\left(S^{4}, B G\right)$ is homotopy equivalent to $\Omega_{0}^{3} G$, the component of $\Omega^{3} G$ containing the basepoint. Putting all this together, for each $k \in \mathbb{Z}$, there is a homotopy fibration sequence

$$
G \stackrel{\partial_{k}}{\longrightarrow} \Omega_{0}^{3} G \longrightarrow B \mathcal{G}_{k} \stackrel{\text { ev }}{\longrightarrow} B G
$$

where $\partial_{k}$ is the fibration connecting map.

Since the homotopy fiber of $\partial_{k}$ is $\mathcal{G}_{k}(G)$, the key to understanding the homotopy type of $\mathcal{G}_{k}(G)$ is to understand $G, \Omega_{0}^{3} G$, and the map $\partial_{k}$ relating them. We begin with the homotopy type of $G$. Let $p$ be an odd prime, and

Kyoto Journal of Mathematics, Vol. 54, No. 3 (2014), 679-691

DOI 10.1215/21562261-2693487, (C) 2014 by Kyoto University

Received December 19, 2012. Revised July 25, 2013. Accepted August 19, 2013.

2010 Mathematics Subject Classification: Primary 55P35; Secondary 54C35, 81T13. 
localize all spaces and maps at $p$. Unless otherwise indicated, homology will be taken with mod- $p$ coefficients. Recall that any simply connected finite $H$ space $X$ is rationally homotopy equivalent to a product of odd-dimensional spheres, $X \simeq_{(0)} \prod_{i=1}^{l} S^{2 n_{i}+1}$. We say that the type of $X$ is $\left\{n_{1}, \ldots, n_{l}\right\}$. The Lie group $G$ is $p$-torsion free if there is no $p$-torsion in $H^{*}(G ; \mathbb{Z})$. As $p$ is odd, it is known that $G$ is $p$-torsion free except for $F_{4}, E_{6}, E_{7}, E_{8}$ at $p=3$ and $E_{8}$ at $p=5$. Mimura, Nishida, and Toda [MNT] showed that if $G$ is $p$ torsion free and of type $\left\{n_{1}, \ldots, n_{l}\right\}$, then there is a $p$-local homotopy equivalence $G \simeq \prod_{i=1}^{p-1} B_{i}$, where the type of $B_{i}$ consists of all those entries $n$ in the type of $G$ satisfying $n \equiv i \bmod (p-1)$. Note that if no entry $n$ in the type of $G$ satisfies $n \equiv i \bmod (p-1)$, then $B_{i}$ is a point. For example, if $G=\operatorname{Sp}(n)$, then $B_{i} \simeq *$ for all even integers $i$.

In [KKT] it was shown that for the same Lie groups $G$, the gauge groups $\mathcal{G}_{k}(G)$ also decompose into a product of $p-1$ factors which depend on the decomposition of $G$. Specifically, there is a homotopy commutative diagram of fibrations

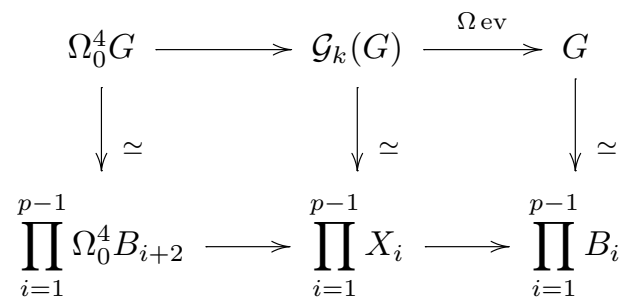

where the bottom row is a product of homotopy fibrations $\Omega_{0}^{4} B_{i+2} \longrightarrow X_{i} \longrightarrow B_{i}$, by $\Omega_{0}^{4} Y$ we mean the component of $\Omega^{4} Y$ containing the basepoint, and if $i=p-2$ or $i=p-1$, then by $i+2$ we mean 1 or 2 , respectively. In this decomposition for $\mathcal{G}_{k}(G)$ it is not clear when the fibrations for $X_{i}$ split. However, more is known if $G$ is a matrix group and the rank of $G$ is low with respect to $p$. The cases involved are (i) $G=\mathrm{SU}(n)$ and $n \leq(p-1)(p-2)+1$, (ii) $G=\operatorname{Sp}(n)$ and $2 n \leq$ $(p-1)(p-2)$, (iii) $G=\operatorname{Spin}(2 n+1)$ and $2 n \leq(p-1)(p-2)$, and (iv) $G=\operatorname{Spin}(2 n)$ and $2(n-1) \leq(p-1)(p-2)$. For such a $G$, an analysis of the map $G \stackrel{\partial_{k}}{\longrightarrow} \Omega_{0}^{3} G$ in [KKT], reformulating an earlier analysis in [T1], shows that $p-3$ of the fibrations $\Omega_{0}^{4} B_{i+2} \longrightarrow X_{i} \longrightarrow B_{i}$ split while the other two may or may not.

In this paper we use a factorization of $\partial_{k}$ arising from the study of moduli spaces of instantons to show that if $G$ is a matrix group of any rank, then $p-3$ of the fibrations for $X_{i}$ split while the remaining two may not. To describe the decompositions precisely we require some notation. The type of $\mathrm{SU}(n)$ is $\{1,2, \ldots, n-1\}$. Let $B_{u}, B_{v}$ be the factors of $\mathrm{SU}(n) \simeq \prod_{i=1}^{p-1} B_{i}$ with the property that $n-2$ and $n-1$ appear in the type of $B_{u}$ and $B_{v}$, respectively. Note that $u=v-1$. The type of $\operatorname{Sp}(n)$ is $\{1,3, \ldots, 2 n-1\}$. Let $B_{w}$ be the factor of $\operatorname{Sp}(n) \simeq$ $\prod_{i=1}^{p-1} B_{i}$ with the property that $2 n-1$ appears in the type of $B_{w}$. It is worth keeping in mind throughout that in the $\operatorname{Sp}(n)$-case the space $B_{i}$ is contractible if $i$ is even. 
THEOREM 1.1

For $n \geq 2$ there are homotopy equivalences

$$
\begin{aligned}
& \mathcal{G}_{k}(\mathrm{SU}(n)) \simeq \prod_{\substack{i=1 \\
i \neq u, v}}^{p-1}\left(B_{i} \times \Omega_{0}^{4} B_{i+2}\right) \times X_{u} \times X_{v}, \\
& \mathcal{G}_{k}(\mathrm{Sp}(n)) \simeq \prod_{\substack{i=1 \\
i \neq w}}^{p-1}\left(B_{i} \times \Omega_{0}^{4} B_{i+2}\right) \times X_{w},
\end{aligned}
$$

where for $t \in\{u, v, w\}$ there is a homotopy fibration $\Omega_{0}^{4} B_{t+2} \longrightarrow X_{t} \longrightarrow B_{t}$.

For $\operatorname{Spin}(n)$, in [KK] and [T1] odd primary homotopy equivalences for $\mathcal{G}_{k}(\operatorname{Spin}(n))$ were established which are analogous to Harris's $[\mathrm{H}]$ odd primary homotopy equivalences $\operatorname{Spin}(2 n+1) \simeq \operatorname{Sp}(n)$ and $\operatorname{Spin}(2 n) \simeq \operatorname{Spin}(2 n-1) \times S^{2 n-1}$. We have the following: (i) for $p \geq 3$ and $k \in \mathbb{Z}$, there is a homotopy equivalence $\mathcal{G}_{k}(\operatorname{Spin}(2 n+1)) \simeq \mathcal{G}_{k}(\operatorname{Sp}(n))$, (ii) for $p \geq 5$ or $p=3$ and $k$ a multiple of 3 , there is a homotopy equivalence $\mathcal{G}_{k}(\operatorname{Spin}(2 n)) \simeq \mathcal{G}_{k}(\operatorname{Spin}(2 n-1)) \times S^{2 n-1} \times \Omega^{4} S^{2 n-1}$, and (iii) an exceptional case proved in [KK], if $p=3$ and $k$ is not a multiple of 3 , then $\mathcal{G}_{k}(\operatorname{Spin}(2 n)) \simeq \mathcal{G}_{k}(\operatorname{Spin}(2 n-1)) \times X$ where $X$ does not split as a product of $S^{2 n-1}$ and $\Omega^{4} S^{2 n-1}$. The full decompositions of $\mathcal{G}_{k}(\operatorname{Spin}(n))$ can then be read off from (i) to (iii) and the decomposition of $\mathcal{G}_{k}(\operatorname{Sp}(n))$ in Theorem 1.1.

\section{Preliminary information on homotopy decompositions of Lie groups}

In this section we give some information on how the decompositions of $\mathrm{SU}(n)$ and $\operatorname{Sp}(n)$ into $p-1$ factors behave with respect to the fibrations $\mathrm{SU}(n-1) \longrightarrow$ $\mathrm{SU}(n) \stackrel{\pi}{\longrightarrow} S^{2 n-1}$ and $\mathrm{Sp}(n-1) \longrightarrow \mathrm{Sp}(n) \stackrel{\pi}{\longrightarrow} S^{4 n-1}$.

First, observe that the type of $\mathrm{SU}(n-1)$ is the same as that of $\mathrm{SU}(n)$ except for the integer $n$. Therefore, in the decompositions of $\mathrm{SU}(n-1)$ and $\mathrm{SU}(n)$ into products of $p-1$ factors, the types of $p-2$ of the factors match. The one exception is the factor $B_{v}$ of $\mathrm{SU}(n)$ which, in the notation from the introduction, has entry $n$ in its type. Suppose that $\mathrm{SU}(n) \simeq\left(\prod_{i=1, i \neq v}^{p-1} B_{i}\right) \times B_{v}$ and $\mathrm{SU}(n-1) \simeq$ $\left(\prod_{i=1, i \neq v}^{p-1} B_{i}^{\prime}\right) \times B_{v}^{\prime}$. Then for $i \neq v$, the factors $B_{i}$ and $B_{i}^{\prime}$ have the same type, and it is easy to see that the composition $B_{i}^{\prime} \hookrightarrow \mathrm{SU}(n-1) \longrightarrow \mathrm{SU}(n) \rightarrow B_{i}$ induces an isomorphism in homology and so is a homotopy equivalence. Therefore $p-2$ of the factors in the homotopy decompositions for $\mathrm{SU}(n)$ and $\mathrm{SU}(n-1)$ are equivalent, and one is different. In the next lemma we make this concrete by proving that the decompositions can be chosen to be compatible and that they behave well with respect to the quotient map $\mathrm{SU}(n) \stackrel{\pi}{\longrightarrow} S^{2 n-1}$. A similar statement also holds for $\operatorname{Sp}(n)$ and $\operatorname{Sp}(n-1)$ and the quotient $\operatorname{map} \operatorname{Sp}(n) \stackrel{\pi}{\longrightarrow} S^{4 n-1}$.

LEMMA 2.1

The homotopy decompositions for $\mathrm{SU}(n-1)$ and $\mathrm{SU}(n)$ (resp., $\mathrm{Sp}(n-1)$ and 
$\mathrm{Sp}(n))$ can be chosen so that there are homotopy commutative diagrams

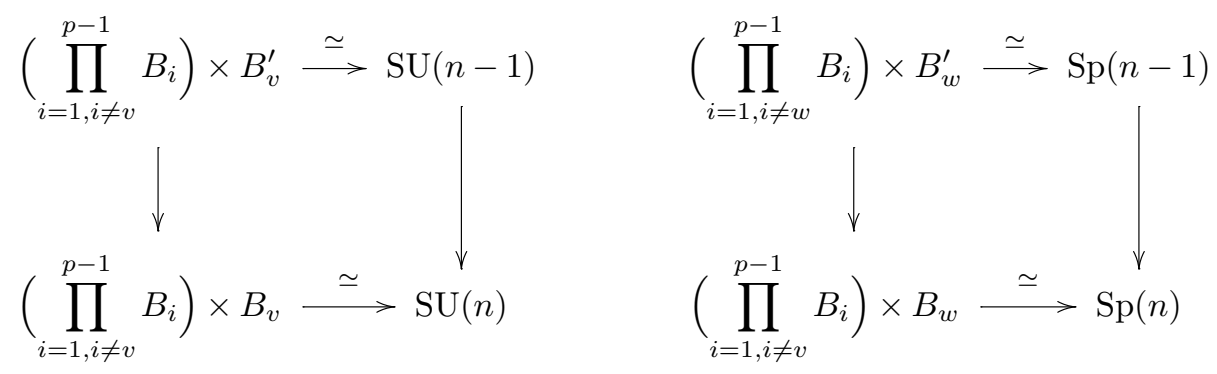

and there are homotopy commutative diagrams
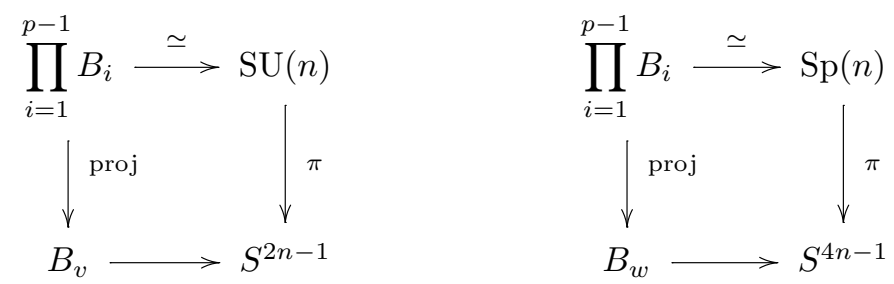

Proof

We prove the $\mathrm{SU}(n)$-case, the argument for $\operatorname{Sp}(n)$ being similar. Start with any choice of homotopy decompositions $\mathrm{SU}(n) \simeq\left(\prod_{i=1, i \neq v}^{p-1} \bar{B}_{i}\right) \times \bar{B}_{v}$ and $\mathrm{SU}(n-1) \simeq$ $\left(\prod_{i=1, i \neq v}^{p-1} \bar{B}_{i}^{\prime}\right) \times \bar{B}_{v}^{\prime}$. We alter the homotopy decompositions as follows. Consider the fibration $\mathrm{SU}(n-1) \longrightarrow \mathrm{SU}(n) \stackrel{\pi}{\longrightarrow} S^{2 n-1}$. Let $B_{v}=\bar{B}_{v}$, and let $g: B_{v} \longrightarrow$ $\mathrm{SU}(n)$ be the composite $B_{v}=\bar{B}_{v} \hookrightarrow\left(\prod_{i=1, i \neq v}^{p-1} \bar{B}_{i}\right) \times \bar{B}_{v} \stackrel{\simeq}{\longrightarrow} \mathrm{SU}(n)$. Define a space $B_{v}^{\prime}$ and maps $g^{\prime}$ and $\kappa$ by the homotopy pullback diagram

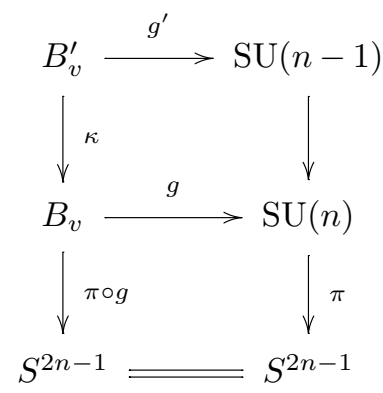

Observe that $B_{v}^{\prime}$ has the same type as $\bar{B}_{v}^{\prime}$, and the composite

$$
B_{v}^{\prime} \stackrel{g^{\prime}}{\longrightarrow} \mathrm{SU}(n-1) \stackrel{\simeq}{\longrightarrow}\left(\prod_{i=1, i \neq v}^{p-1} \bar{B}_{i}^{\prime}\right) \times \bar{B}_{v}^{\prime} \stackrel{\text { proj }}{\longrightarrow} \bar{B}_{v}^{\prime}
$$

induces an isomorphism in homology and so is a homotopy equivalence. 
For $1 \leq i \leq p-1$ and $i \neq v$, let $B_{i}=\bar{B}_{i}^{\prime}$. Let $f^{\prime}$ be the composite

$$
f^{\prime}: \prod_{i=1, i \neq v}^{p-1} B_{i}=\prod_{i=1, i \neq v}^{p-1} \bar{B}_{i}^{\prime} \hookrightarrow\left(\prod_{i=1, i \neq v}^{p-1} \bar{B}_{i}^{\prime}\right) \times \bar{B}_{v}^{\prime} \stackrel{\simeq}{\longrightarrow} \mathrm{SU}(n-1),
$$

and let $f$ be the composite

$$
f: \prod_{i=1, i \neq v}^{p-1} B_{i} \stackrel{f^{\prime}}{\longrightarrow} \mathrm{SU}(n-1) \longrightarrow \mathrm{SU}(n) .
$$

Using the loop multiplications $\mu$ on $\mathrm{SU}(n-1)$ and $\mathrm{SU}(n)$, we therefore obtain a homotopy commutative diagram

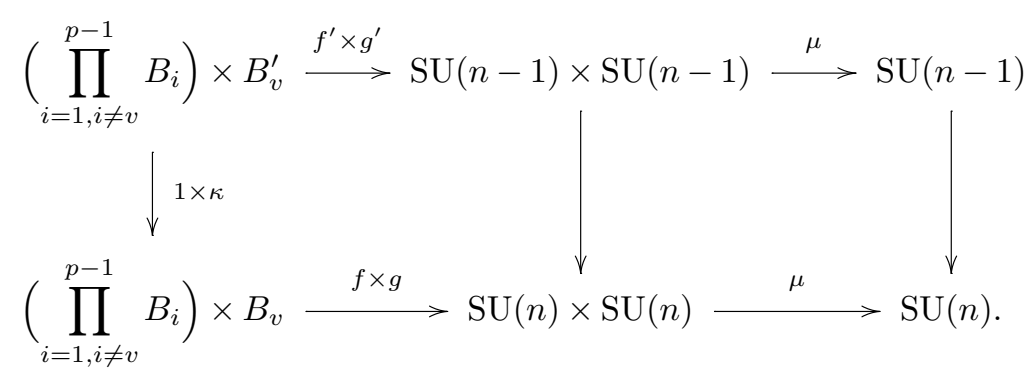

The two rows induce isomorphisms in homology and so are homotopy equivalences. The outer diagram establishes the compatibility of the decompositions of $\mathrm{SU}(n-1)$ and $\mathrm{SU}(n)$ asserted by the first diagram in the statement of the lemma.

For the second diagram involving $\pi$, observe in general that for a homotopy fibration sequence $\Omega B \stackrel{\delta}{\longrightarrow} F \longrightarrow E \longrightarrow B$, the connecting map induces a homotopy action $\theta: F \times \Omega B \longrightarrow F$ with the property that there is a homotopy commutative square

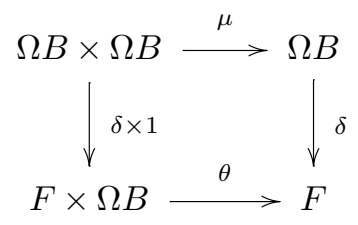

where $\mu$ is the loop multiplication on $\Omega B$.

For $\mathrm{SU}(n)$ there is a homotopy fibration sequence $\mathrm{SU}(n) \stackrel{\pi}{\longrightarrow} S^{2 n-1} \longrightarrow$ $\mathrm{BSU}(n-1) \longrightarrow \mathrm{BSU}(n)$. Using the homotopy equivalence $\mu \circ(f \times g)$ in (3), consider the diagram 


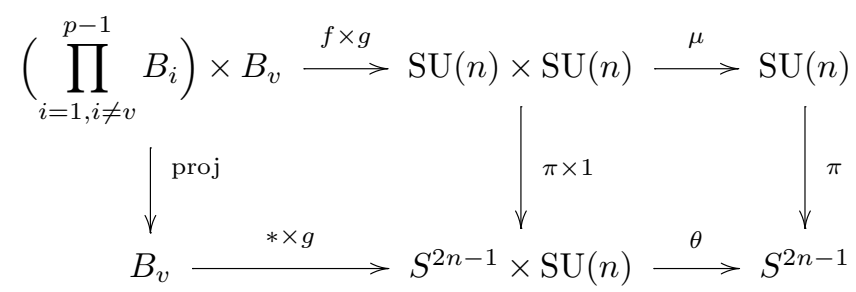

The right square homotopy commutes since $\pi$ is a fibration connecting map and so has a homotopy action. The left square homotopy commutes since, by (3), the map $f$ factors through $\mathrm{SU}(n-1)$. The composition along the top row is a homotopy equivalence. The outer perimeter of the diagram then gives the homotopy commutative square asserted by the lemma.

\section{A factorization of the boundary map $\partial_{k}$}

In this section we factor the boundary map $G \stackrel{\partial_{k}}{\longrightarrow} \Omega_{0}^{3} G$ for $G=\mathrm{SU}(n)$ or $G=$ $\operatorname{Sp}(n)$. We begin with an identification of the triple adjoint of $\partial_{k}$ due to Lang [L]. Let $i: S^{3}=\mathrm{SU}(2) \longrightarrow \mathrm{SU}(n)$ be the standard group homomorphism. We also use $i$ to denote the standard group homomorphism $i: S^{3}=\operatorname{Sp}(1) \longrightarrow \operatorname{Sp}(n)$.

\section{LEMMA 3.1}

The triple adjoint of $\partial_{k}$ is homotopic to the Samelson product $S^{3} \wedge G \stackrel{\langle k \cdot i, 1\rangle}{\longrightarrow} G$ where 1 is the identity map on $G$.

The linearity of the Samelson product immediately implies the following.

\section{COROLLARY 3.2}

There is a homotopy $\partial_{k} \simeq k \circ \partial_{1}$, where $k$ is the $k$ th-power map on $\Omega_{0}^{3} G$.

Corollary 3.2 implies that we need only be concerned about $\partial_{1}$. Lemma 3.1 lets us describe $\partial_{1}$ explicitly, up to homotopy. Notice that an element of $\Omega_{0}^{3} G$ is a basepoint-preserving map $S^{3} \longrightarrow G$ of degree 0 . Define

$$
\theta: G \longrightarrow \Omega_{0}^{3} G
$$

by sending $g$ to the map $\theta_{g}$ which satisfies $\theta_{g}(x)=g x g^{-1} x^{-1}$ for $x \in S^{3}$. By definition, $\theta$ is the triple adjoint of the Samelson product $\langle i, 1\rangle$. In particular, it is continuous. Moreover, by Lemma 3.1, $\partial_{1}$ is homotopic to $\theta$.

On the other hand, when considering moduli spaces of instantons, Boyer, Mann, and Waggoner [BMW] give a factorization of $\theta$ through the quotient of $G$ by the centralizer of $\mathrm{SU}(2)$ in $G$. For $G=\mathrm{SU}(n)$, the centralizer consists of matrices of the form

$$
\left[\begin{array}{lll}
a & 0 & 0 \\
0 & a & 0 \\
0 & 0 & A
\end{array}\right]
$$


where $A \in U(n-2)$ and $\operatorname{det}(A)=a^{-2}$, and for $G=\operatorname{Sp}(n)$ the centralizer consists of matrices of the form

$$
\left[\begin{array}{cc}
a & 0 \\
0 & a A
\end{array}\right]
$$

where $A \in \operatorname{Sp}(n-1)$ and $a \in \mathbb{Z} / 2 \mathbb{Z}$ is in the center of $\operatorname{Sp}(1)$. Note that in the $\mathrm{SU}(n)$-case the centralizer is homotopy equivalent to $\mathrm{SU}(n-2) \times U(1)$ and in the $\operatorname{Sp}(n)$-case the centralizer is homotopy equivalent to $\mathbb{R} P^{4 n-1}$. Boyer, Mann, and Waggoner show that there are commutative diagrams

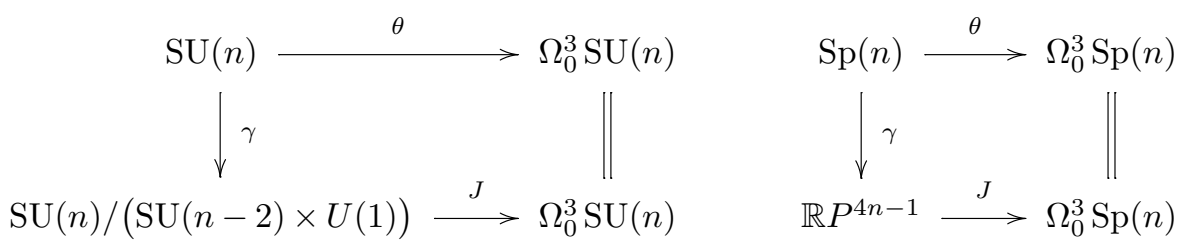

where the maps labeled $\gamma$ are the quotient maps induced by the inclusion of the centralizers, and the maps labeled $J$ have the property that if $C=\mathrm{SU}(n-2) \times$ $U(1)$ or $C=\mathbb{R} P^{4 n-1}$, respectively, then for a coset $g C$ and $x \in \mathrm{SU}(2)$ the map $J$ is defined as $J(g C)(x)=g x g^{-1} x^{-1}$.

Observe that the map $\gamma$ for $\mathrm{SU}(n)$ factors as the composite $\mathrm{SU}(n) \stackrel{\pi}{\longrightarrow}$ $\mathrm{SU}(n) / \mathrm{SU}(n-2) \longrightarrow \mathrm{SU}(n) /(\mathrm{SU}(n-2) \times U(1))$ where $\pi$ is the standard quotient map. Similarly, the map $\gamma$ for $\operatorname{Sp}(n)$ factors as the composite $\operatorname{Sp}(n) \stackrel{\pi}{\longrightarrow}$ $\operatorname{Sp}(n) / \operatorname{Sp}(n-1) \cong S^{4 n-1} \longrightarrow \mathbb{R} P^{4 n-1}$. Thus, weakening the factorizations of $\theta$ through $\gamma$ to $\theta$ through $\pi$, and using the homotopies $\theta \simeq \partial_{1}$ and $\partial_{k} \simeq k \circ \partial_{1}$, we obtain the following.

PROPOSITION 3.3

There are homotopy commutative diagrams
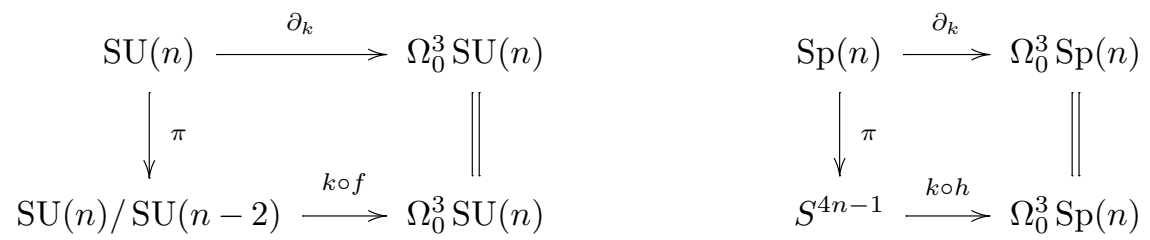

for some maps $f$ and $h$.

\section{REMARK 3.4}

As pointed out by the referee, the factorizations in Proposition 3.3 arising from [BMW] were in fact obtained much earlier by Bott [B].

In Section 4 we will use the factorization in Proposition 3.3 to establish the homotopy decompositions in Theorem 1.1. To go further and consider the homotopy types of the factors $X_{u}, X_{v}$, and $X_{w}$, it would be useful to know the order of $\partial_{k}$. This is known only for the special cases of $\mathrm{SU}(2)$ (see [Ko]), $\mathrm{SU}(3)$ 
(see [HK]), and $\operatorname{Sp}(2)$ (see [T2]). More is known if the prime 2 is excluded: by $[\mathrm{KKT}]$ the odd primary components of the order of $\partial_{k}$ are known for $\mathrm{SU}(n)$ when $n-1 \leq(p-1)(p-2)$ and for $\operatorname{Sp}(n)$ when $2 n-1 \leq(p-1)(p-2)$. In general, Proposition 3.3 in the $\operatorname{Sp}(n)$-case shows that the order of $\partial_{k}$ divides the order of $k \circ h$, where $h$ is an element of $\pi_{4 n+2}(\operatorname{Sp}(n))$, which is isomorphic to $\mathbb{Z} /(2 n+1) ! \mathbb{Z}$ if $n$ is even and to $\mathbb{Z} / 2(2 n+1) ! \mathbb{Z}$ if $n$ is odd. It is likely that the order of $\partial_{k}$ is much less than the order of $k \circ h$. In the $\mathrm{SU}(n)$-case, the order of $f$ is unclear, but its restriction to the bottom cell $S^{2 n-3}$ is an element of $\pi_{2 n}(\mathrm{SU}(n)) \cong \mathbb{Z} / n ! \mathbb{Z}$. Further, the second author's analysis of the map $\theta$ in [Ki] shows that there is a homotopy commutative diagram

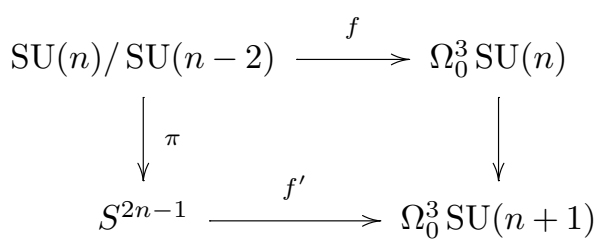

for some map $f^{\prime}$, and $f^{\prime}$ is an element of $\pi_{2 n+2}(\mathrm{SU}(n+1)) \cong \mathbb{Z} /(n+1) ! \mathbb{Z}$. Again, it is likely that the order of $f$ is much less than $(n+1)$ !.

\section{Homotopy decompositions}

In this section we establish the homotopy decompositions in Theorem 1.1. We first require a lemma. A homotopy fibration $F \stackrel{i}{\longrightarrow} E \stackrel{\pi}{\longrightarrow} B$ is said to be a homotopy retract of a homotopy fibration $F^{\prime} \stackrel{i^{\prime}}{\longrightarrow} E^{\prime} \stackrel{\pi^{\prime}}{\longrightarrow} B^{\prime}$ if there is a homotopy commutative diagram

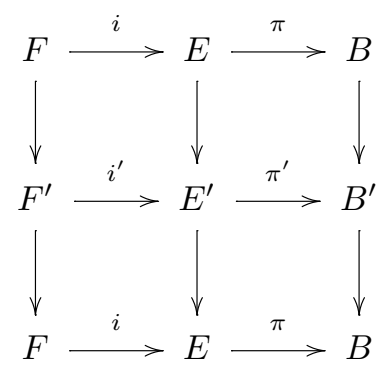

where all three vertical composites are homotopy equivalences. As well, a homotopy fibration $F \stackrel{i}{\longrightarrow} E \stackrel{\pi}{\longrightarrow} B$ is an $H$-fibration if all spaces are $H$-spaces and both $i$ and $\pi$ are $H$-maps.

LEMMA 4.1

Suppose that a homotopy fibration $F \stackrel{i}{\longrightarrow} E \stackrel{\pi}{\longrightarrow} B$ is a homotopy retract of an $H$-fibration $F^{\prime} \stackrel{i^{\prime}}{\longrightarrow} E^{\prime} \stackrel{\pi^{\prime}}{\longrightarrow} B^{\prime}$. If the map $E \stackrel{\pi}{\longrightarrow} B$ has a section, then $E \simeq$ $B \times F$. 
Proof

Let $s: B \longrightarrow E$ be a section for the map $E \stackrel{\pi}{\longrightarrow} B$. Let $\bar{s}$ be the composite $\bar{s}: B \stackrel{s}{\longrightarrow}$ $E \longrightarrow E^{\prime}$, and let $\bar{i}$ be the composite $\bar{i}: F \stackrel{i}{\longrightarrow} E \longrightarrow E^{\prime}$. Consider the diagram

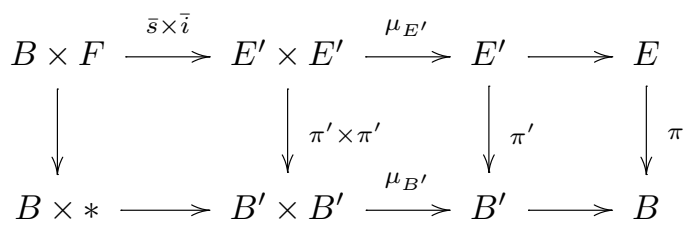

where $\mu_{E^{\prime}}$ and $\mu_{B^{\prime}}$ are the multiplications on $E^{\prime}$ and $B^{\prime}$, respectively. The lefthand square homotopy commutes since, by (4), $\bar{i}$ factors through $i^{\prime}$. The middle square homotopy commutes since $\pi^{\prime}$ is an $H$-map, and the right-hand square homotopy commutes by (4). Let $\epsilon: B \times F \longrightarrow E$ be the composite along the top row. Then the homotopy commutativity of this diagram implies that $\pi \circ \epsilon$ is homotopic to the projection onto $B$, and the definition of $\bar{i}$ implies that the restriction of $\epsilon$ to $F$ is $i$. Thus we obtain a homotopy fibration diagram

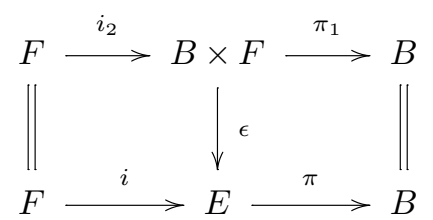

where $i_{2}$ is the inclusion of the second factor and $\pi_{1}$ is the projection to the first. Thus $\epsilon$ is a homotopy pullback of $\pi$ and the identity map on $B$, and so is a homotopy equivalence.

Proof of Theorem 1.1

First consider the case of $\mathrm{SU}(n)$. By (2), there is a homotopy commutative square

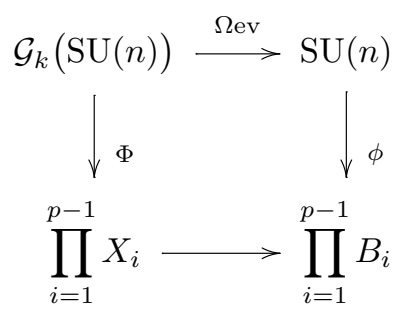

where $\Phi$ and $\phi$ are homotopy equivalences and the map along the bottom is a product of maps $X_{i} \longrightarrow B_{i}$. Consider the homotopy fibration $\mathcal{G}_{k}(\mathrm{SU}(n)) \stackrel{\Omega \mathrm{ev}}{\longrightarrow}$ $\mathrm{SU}(n) \stackrel{\partial_{k}}{\longrightarrow} \Omega_{0}^{3} \mathrm{SU}(n)$. By Proposition 3.3, $\partial_{k}$ factors through the quotient map $\mathrm{SU}(n) \stackrel{\pi}{\longrightarrow} \mathrm{SU}(n) / \mathrm{SU}(n-2)$. Consequently, the composition $\mathrm{SU}(n-2) \longrightarrow$ $\mathrm{SU}(n) \stackrel{\partial_{k}}{\longrightarrow} \Omega_{0}^{3} \mathrm{SU}(n)$ is null homotopic, implying that there is a lift 


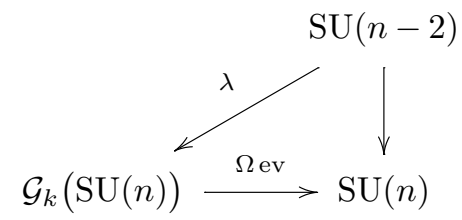

for some map $\lambda$. By Lemma 2.1, there is a homotopy equivalence $\varphi: \prod_{i=1}^{p-1} B_{i} \longrightarrow$ $\mathrm{SU}(n)$ with the property that the restriction of $\varphi$ to $\prod_{i=1, i \notin\{u, v\}}^{p-1} B_{i}$ factors through $\mathrm{SU}(n-2)$. Thus we obtain a homotopy commutative diagram

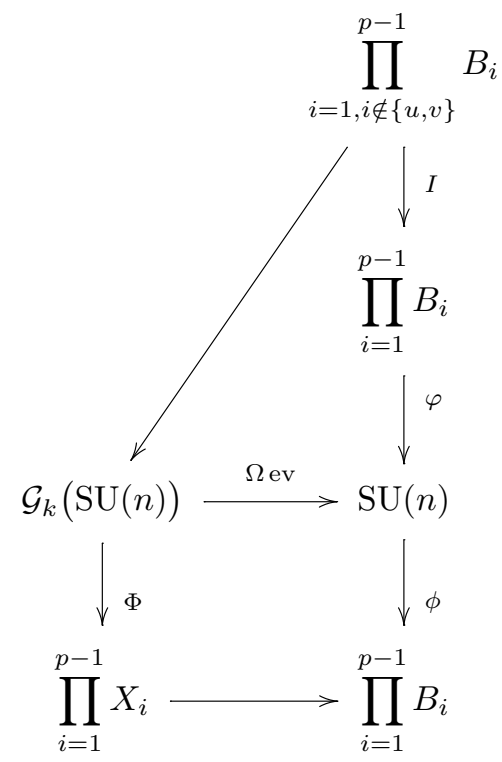

where $I$ is the inclusion. Note that $\varphi$ may not be the inverse of $\phi$, but $\phi \circ \varphi$ is a homotopy equivalence. Thus $(\phi \circ \varphi)^{*}$ induces an algebra isomorphism in cohomology. Since no two algebra generators in $H^{*}\left(\prod_{i=1}^{p-1} B_{i}\right)$ occur in the same dimension, it therefore must be the case that for each $1 \leq t \leq p-1$ the composite $B_{t} \stackrel{\text { incl }}{\longrightarrow} \prod_{i=1}^{p-1} B_{i} \stackrel{\phi \circ \varphi}{\longrightarrow} \prod_{i=1}^{p-1} B_{i} \stackrel{\text { proj }}{\longrightarrow} B_{t}$ induces an isomorphism on algebra generators in cohomology, and therefore induces an isomorphism in cohomology, and so is a homotopy equivalence. Consequently, for each $i \notin\{u, v\}$, the homotopy fibration $\Omega_{0}^{4} B_{i+2} \longrightarrow X_{i} \longrightarrow B_{i}$ has a section $B_{i} \longrightarrow X_{i}$. By (2), the homotopy fibration $\Omega_{0}^{4} B_{i+2} \longrightarrow X_{i} \longrightarrow B_{i}$ is a retract of the $H$-fibration $\Omega_{0}^{4} \mathrm{SU}(n) \longrightarrow$ $\mathcal{G}_{k}(\mathrm{SU}(n)) \stackrel{\Omega \text { ev }}{\longrightarrow} \mathrm{SU}(n)$. So by Lemma 4.1 , the existence of a section for the map $X_{i} \longrightarrow B_{i}$ implies that there is a homotopy equivalence $X_{i} \simeq B_{i} \times \Omega_{0}^{4} B_{i+2}$. Hence

$$
\mathcal{G}_{k}(\mathrm{SU}(n)) \simeq \prod_{i=1, i \notin\{u, v\}}^{p-1}\left(B_{i} \times \Omega_{0}^{4} B_{i+2}\right) \times X_{u} \times X_{v} .
$$


The argument for $\operatorname{Sp}(n)$ is exactly the same, using the fact that from Proposition 3.3 that the map $\operatorname{Sp}(n) \stackrel{\partial_{k}}{\longrightarrow} \Omega_{0}^{3} \mathrm{Sp}(n)$ factors through the quotient map $\operatorname{Sp}(n) \stackrel{\pi}{\longrightarrow} S^{4 n-1}$.

\section{An alternative proof in the $\operatorname{Sp}(n)$-case}

In the $\mathrm{Sp}(n)$-case, there is an alternative proof of the homotopy decomposition in Theorem 1.1 which is independent of the decomposition of $\mathcal{G}_{k}(\operatorname{Sp}(n))$ in [KKT].

Alternative proof of Theorem 1.1 for $G=\operatorname{Sp}(n)$

Recall that the homotopy equivalence $e: \operatorname{Sp}(n) \stackrel{\simeq}{\longrightarrow} \prod_{i=1}^{p-1} B_{i}$ of Lemma 2.1 has a distinguished factor $B_{w}$ with the property that $2 n-1$ appears in its type. Now consider the map $S^{4 n-1} \stackrel{h}{\longrightarrow} \Omega_{0}^{3} \mathrm{Sp}(n)$ appearing in the diagram in Proposition 3.3. This map represents a class in $\pi_{4 n+2}(\operatorname{Sp}(n))$. This homotopy group has a single generator, which is inherited by a single factor $B_{i}$ under the homotopy equivalence $e$. The homotopy group calculations for the $B_{i}$ 's in [MNT] imply that $\pi_{4 n+2}\left(B_{i}\right)=0$ unless $i=w+2$. Thus $h$ factors as a composite $S^{4 n-1} \stackrel{h^{\prime}}{\longrightarrow}$ $\Omega_{0}^{3} B_{w+2} \hookrightarrow \prod_{i=1}^{p-1} \Omega_{0}^{3} B_{i} \stackrel{\Omega^{3} e^{-1}}{\longrightarrow} \Omega_{0}^{3} \operatorname{Sp}(n)$.

Let $g_{k}$ be the composite

$$
g_{k}: B_{w} \longrightarrow S^{4 n-1} \stackrel{k \circ h^{\prime}}{\longrightarrow} \Omega_{0}^{3} B_{w+2},
$$

and let $\bar{g}_{k}$ be the composite

$$
\bar{g}_{k}: \prod_{i=1}^{p-1} B_{i} \stackrel{\text { proj }}{\longrightarrow} B_{w} \stackrel{g_{k}}{\longrightarrow} \Omega_{0}^{3} B_{w+2} \hookrightarrow \prod_{i=1}^{p-1} \Omega_{0}^{3} B_{i} .
$$

Consider the diagram

$$
\begin{aligned}
& \prod_{i=1}^{p-1} B_{i} \stackrel{e^{-1}}{\longrightarrow} \operatorname{Sp}(n) \stackrel{\partial_{k}}{\longrightarrow} \Omega_{0}^{3} \operatorname{Sp}(n) \\
& \qquad \begin{array}{l|l}
\text { proj } \\
\downarrow
\end{array} \\
& B_{w} \stackrel{\Downarrow}{\longrightarrow} S^{4 n-1} \stackrel{k \circ h}{\longrightarrow} \Omega_{0}^{3} \operatorname{Sp}(n) \stackrel{\Omega^{3} e}{\longrightarrow}\left(\prod_{i=1, i \neq w+2}^{p-1} \Omega_{0}^{3} B_{i}\right) \times \Omega_{0}^{3} B_{w+2} .
\end{aligned}
$$

The left-hand square homotopy commutes by Lemma 2.1, and the middle square homotopy commutes by Proposition 3.3. The factorization of $h$ described above implies that the composite along the lower direction of this diagram is the definition of $\bar{g}_{k}$. Thus there is a homotopy commutative diagram 


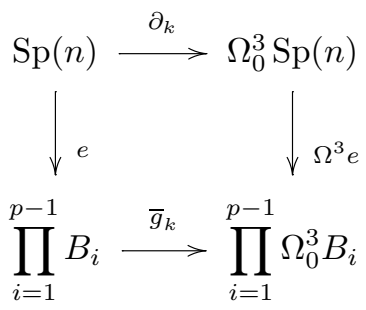

The homotopy fiber of $\partial_{k}$ is $\mathcal{G}_{k}(\operatorname{Sp}(n))$. By definition, $\bar{g}_{k}$ factors through a projection onto the single factor $B_{w}$ and the inclusion of the one factor $\Omega_{0}^{3} B_{w+2}$. So the homotopy fiber of $\bar{g}_{k}$ is $\prod_{i=1, i \neq w}^{p-1}\left(B_{i} \times \Omega_{0}^{4} B_{i+2}\right) \times X_{w}$, where $X_{w}$ is the homotopy fiber of $g_{k}$. The homotopy commutativity of (5) induces a map of fibers, which is a homotopy equivalence since both $e$ and $\Omega^{3} e$ are. Hence $\mathcal{G}_{k}(\operatorname{Sp}(n)) \simeq \prod_{i=1, i \neq w}^{p-1}\left(B_{i} \times \Omega_{0}^{4} B_{i+2}\right) \times X_{w}$.

It is unclear whether the same method works for the $\mathrm{SU}(n)$-case. For example, it is not clear whether there is an analogue of Lemma 2.1 that produces a homotopy equivalence $\mathrm{SU}(n) \simeq \prod_{i=1}^{p-1} B_{i}$ which is sufficiently well behaved with respect to the quotient map $\mathrm{SU}(n) \longrightarrow \mathrm{SU}(n) / \mathrm{SU}(n-2)$.

Acknowledgment. The authors would like to thank the referees for an observant reading of the paper and for offering several suggestions for improvements.

\section{References}

[B] R. Bott, A note on the Samelson product in the classical groups, Comment. Math. Helv. 34 (1960), 249-256. MR 0123330.

[BMW] C. P. Boyer, B. M. Mann, and D. Waggonner, On the homology of SU(n) instantons, Trans. Amer. Math. Soc. 323 (1991), no. 2, 529-561.

MR 1034658. DOI 10.2307/2001544.

[G] D. H. Gottlieb, Applications of bundle map theory, Trans. Amer. Math. Soc. 171 (1972), 23-50. MR 0309111.

[HK] H. Hamanaka and A. Kono, Unstable $K^{1}$-group and homotopy type of certain gauge groups, Proc. Roy. Soc. Edinburgh Sect. A 136 (2006), 149-155. MR 2217512. DOI 10.1017/S0308210500004480.

[H] B. Harris, On the homotopy groups of the classical groups, Ann. of Math. (2) 74 (1961), 407-413. MR 0131278.

[Ki] D. Kishimoto, Generating varieties, Bott periodicity and instantons, Topology Appl. 157 (2010), 657-668. MR 2577499.

DOI 10.1016/j.topol.2009.11.007.

[KK] D. Kishimoto and A. Kono, Note on mod-p decompositions of gauge groups, Proc. Japan Acad. Ser. A. Math. Sci. 86 (2010), 15-17. MR 2598819. 
[KKT] D. Kishimoto, A. Kono, and M. Tsutaya, Mod-p decompositions of gauge groups, Algebr. Geom. Topol. 13 (2013), 1757-1778. MR 3071142. DOI 10.2140/agt.2013.13.1757.

[Ko] A. Kono, A note on the homotopy type of certain gauge groups, Proc. Roy. Soc. Edinburgh Sect. A 117 (1991), 295-297. MR 1103296. DOI 10.1017/S0308210500024732.

[L] G. E. Lang, The evaluation map and EHP sequences, Pacific J. Math. 44 (1973), 201-210. MR 0341484.

[MNT] M. Mimura, G. Nishida, and H. Toda, Mod-p decomposition of compact Lie groups, Publ. Res. Inst. Math. Sci. 13 (1977/78), 627-680. MR 0478187.

[T1] S. D. Theriault, Odd primary decompositions of gauge groups, Algebr. Geom. Topol. 10 (2010), 535-564. MR 2602840. DOI 10.2140/agt.2010.10.535.

$[\mathrm{T} 2]$ , The homotopy types of $\mathrm{Sp}(2)$-gauge groups, Kyoto J. Math. 50 2010, 591-605. MR 2723863. DOI 10.1215/0023608X-2010-005.

Kishimoto: Department of Mathematics, Kyoto University, Kyoto 606-8502, Japan; kishi@math.kyoto-u.ac.jp

Kono: Faculty of Science and Engineering, Doshisha University, Kyoto 610-0321, Japan; akono@mail.doshisha.ac.jp

Theriault: School of Mathematics, University of Southampton, Southampton SO17 1BJ, United Kingdom; s.d.theriault@soton.ac.uk 